\title{
Monte Carlo uncertainty propagation approaches in ADS burn-up calculations
}

\author{
C.J. Díez ，O. Cabellos ，D. Rochman , A.J. Koning , J.S. Martínez
}

\begin{abstract}
A B S T R A C T
In activation calculations, there are several approaches to quantify uncertainties: deterministic by means of sensitivity analysis, and stochastic by means of Monte Carlo. Here, two different Monte Carlo approaches for nuclear data uncertainty are presented: the first one is the Total Monte Carlo (TMC). The second one is by means of a Monte Carlo sampling of the covariance information included in the nuclear data libraries to propagate these uncertainties throughout the activation calculations. This last approach is what we named Covariance Uncertainty Propagation, CUP.

This work presents both approaches and their differences. Also, they are compared by means of an activation calculation, where the cross-section uncertainties of ${ }^{239} \mathrm{Pu}$ and ${ }^{241} \mathrm{Pu}$ are propagated in an ADS activation calculation.
\end{abstract}

\section{Introduction}

After Fukushima's accident, nuclear safety is in the spotlight. New stress assessments are on going in every nuclear power plant, just to check their safe performance and review their safety margins are properly set.

These safety margins guarantee the safe performance of the facility during and after an accident. These safety margins are designed to take into account all the uncertainties throughout the designing stage, such as the uncertainties on the engineering designs, model uncertainties, data uncertainties used on the design, build-up and operation.

Nuclear data uncertainties have an impact on the safety margins as they are used in engineering calculations such as core neutronics or material activation. In this case, we focus on activation calculations and their associated uncertainties due to the nuclear data uncertainties. There are mainly three different kinds of nuclear data: decay data, fission yields and cross-section data.

In order to measure the effect of these uncertainty data on activation calculations, uncertainty propagation calculations can be done. There are several approaches to perform uncertainty propagation calculations. One of them is the well-known approach of the sensitivity analysis (Cacuci, 2003), which uses a first-order Taylor series approximation of the response function. Two other approaches, that we will talk about later, are Monte Carlo (MC) approaches based on randomizing the parameters of the response function, using Probability Density Functions (PDFs) to sample. Then, calculating the response function with a large enough amount of samples, the uncertainty of the response function can be calculated.

One of these two MC approaches for uncertainty propagation is a novel approach named Total Monte Carlo (TMC) (Koning and Rochman, 2008; Rochman et al., 2009, 2011; Koning and Rochman, 2011). It is based on randomizing the nuclear model parameters of the TALYS code to generate random nuclear data within the uncertainties of the experimental data. With them, response functions can be calculated and, after a statistical analysis, its uncertainty too.

The other approach of carrying out uncertainty propagation is what we call "the Covariance Uncertainty Propagation (CUP)" described in this paper. Briefly, instead of using a nuclear model code for generating random nuclear data files, uncertainty information of the nuclear data library is used to obtain random nuclear data files. Hence, the response functions and their uncertainties can be calculated using these files.

When these two approaches of performing $\mathrm{MC}$ nuclear data uncertainty propagation are compared, a few questions arise: Are both of them equivalent? In case they are not equivalent, which are the differences between them? Which are the sources of these differences?

Therefore, the aim of this paper is to answer the questions above for cross-section uncertainties only. Both approaches are implemented and compared for an activation calculation with 
uncertainty propagation for two isotopes: ${ }^{239} \mathrm{Pu}$ and ${ }^{241} \mathrm{Pu}$. A complete set of cross-sections for all isotopes is used: EAF-2010 (Sublet et al., 2010). Then, the random cross-sections of a single isotope, at a time, are merged with this complete library. The random files for the TMC approach come from TENDL-2010 (Koning and Rochman, 2010) and the uncertainty data of the cross-sections are stored as covariance matrices produced by the TMC random files.

\section{Monte Carlo uncertainty propagation}

In this Section 2, the two approaches of MC uncertainty propagation are presented and applied to activation calculations: Total Monte Carlo (TMC) and Covariance Uncertainty Propagation (CUP).

\subsection{Total Monte Carlo, TMC}

The main aim of the TMC approach is to simulate consequences of uncertainties in semi-microscopic nuclear physics on nuclear design without any limitation in between. Then, Nuclear Data (ND) uncertainties can be propagated in any kind of calculation without any approximation. This approach is based on the TALYS software package (Koning et al., 2009), which contains different codes. A flowchart of the system is presented in Fig. 1 which shows that by looping over the entire process of basic nuclear physics, data file production, data file processing and applied calculations, a natural statistical approach towards uncertainty propagation can be obtained.

TASMAN uses central value parameters obtained from a best fit to experimental cross-sections and angular distributions. The uncertainties on these parameters are obtained after randomly sampling the parameters and a check whether the results are inside the experimental data uncertainties (i.e. retrieved from EXFOR database (Rugama et al., 11 May 2005-14 May 2005)). Then, the process of assigning uncertainties to the nuclear data parameters proceeds in two steps. First, suggested parameter uncertainties are used to start the sampling of cross-sections. Using these results, it is checked which ones are inside the experimental data. Second, this information is fed back to reduce or increase the parameter uncertainty, producing uncertainties that properly reproduce the experimental data and their dispersions. The PDF can be chosen among equiprobable, Normal or other. In principle, with the least information available, the equiprobable is chosen; otherwise, the Normal PDF is considered.

It is important to remark that nuclear model parameters are independently randomized. But, because there are several constraints on the variation between different cross-sections inside the nuclear models, different cross-sections are highly correlated. The main constraint is the total cross-section whose value is calculated using one nuclear model and then is fed to the other nuclear models that calculate differential cross-sections such as $(n, \gamma),(n$, $2 n$ ) or ( $n$, inel) reactions. That means that the sum of all these differential cross-sections cannot exceed the total cross-section value. Also, there are high correlation between different energies because of the nuclear model stiffness.

\subsection{Covariance Uncertainty Propagation, CUP}

In the last years, nuclear data libraries such as TENDL-2010 and ENDF/B.VII.0 (Chadwick et al., 2006) have included information of cross-section uncertainties. This information can be used to perform uncertainty propagation calculations. But for MC approaches, this information should be used in a different way than sensitivity analysis.

This approach uses the information of the mean value, the variance and covariance included in nuclear data libraries to generate random cross-section files. Choosing a PDF, cross-section values are sampled using the mean value and std.dev. provided by the ND library. Then, a random sampling of the cross-sections is performed and a large amount of random nuclear data libraries is produced. This amount of random cross-section libraries can be used to carry out uncertainty propagation calculations. After running the application calculation with all the random cross-section libraries, a statistical analysis of the response variables (such as decay heat) can be done. It provides the mean value and its std.dev. (or rel.std.dev.) as a measurement of uncertainty.

Cross-section uncertainties in libraries have to be processed to be used. For this purpose, the NJOY code (MacFarlane and Muir, 1994 ) is used as processing code. It handles the information in MF32 and MF33 of ENDF-format to produce a desired group-wise energy structure of the cross-sections and their uncertainties.

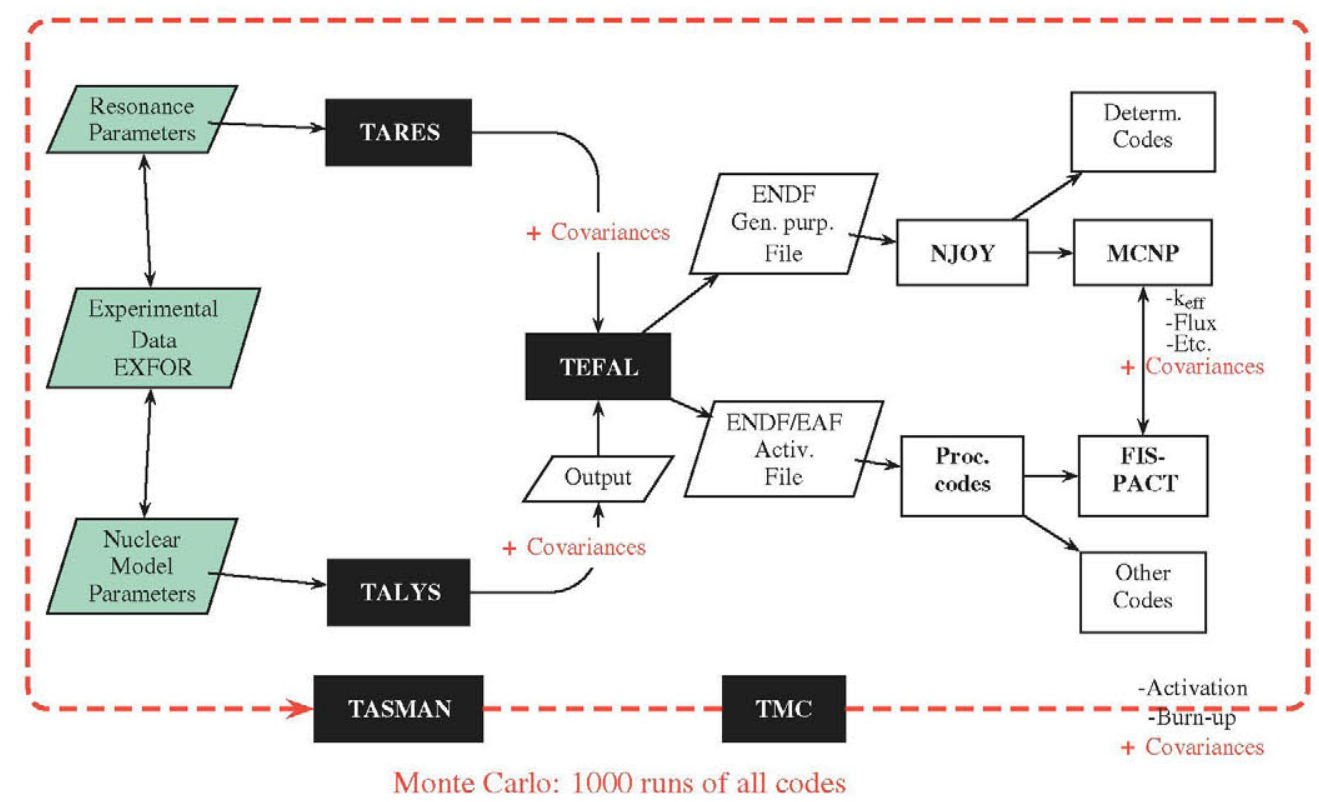

Fig. 1. Flowchart of Total Monte Carlo calculations, involving the four codes from the TALYS code system, processing and transport/reactor codes. 


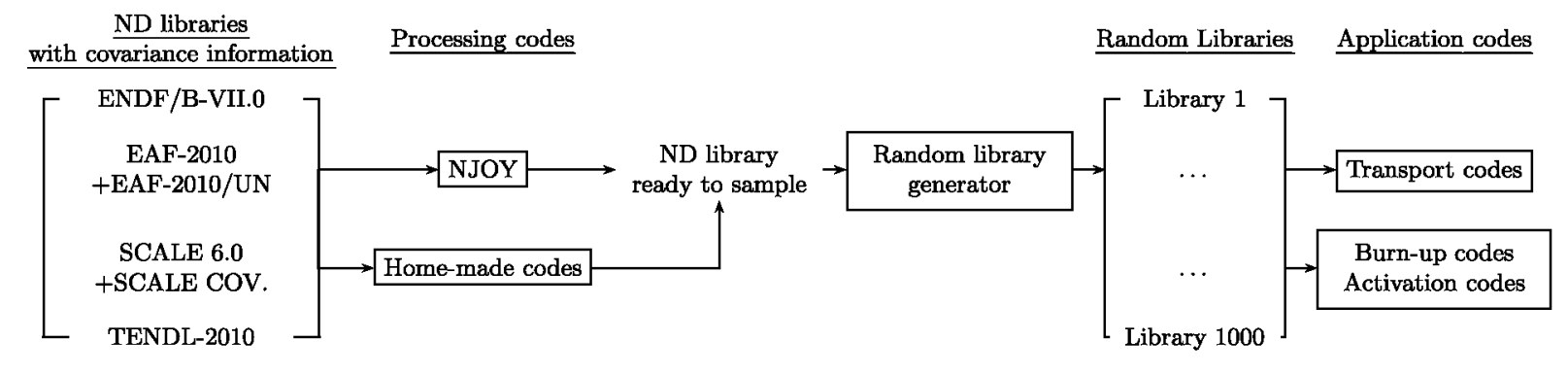

Fig. 2. Flowchart of covariance uncertainty propagation calculations.

Throughout this stage, the spectrum of the studied application is required to process this information. After this processing step, cross-sections are ready to be sampled using their uncertainties (and their covariance matrix). If any cross-correlation among reactions exists, the Cholesky Decomposition of the covariance matrix is performed, as done before in Cabellos et al. (2011) in order to generate correlated cross section values. Letting $T$ verifies $\Sigma=T^{T} T$, where $\Sigma$ is the covariance matrix, correlated cross section values are obtained using Eq. (1), where $x$ is the correlated cross section vector, $z$ is a vector of identically and independently distributed standard normal deviates and $\mu$ is the mean cross section value vector. Other approaches could be applied as exposed in Gentle (2003).

$x=T^{T} z+\mu$

There are several advanced sampling methods that the CUP approach does not use right now, only the simply "random sampling" (RS) is used.

Before sampling, one assumption should be done in this approach: which PDF is used to sample the uncertainty information. Nuclear data libraries do not provide any information about which kind of PDF is followed by each cross-section. So, it is up to the user to choose one PDF to sample the cross-sections. In this approach, it is assumed that cross-sections follow Normal PDFs.

With each random library, an application calculation can be launched, and thus, the cross-section uncertainties are propagated. Fig. 2 shows an scheme of the methodology for using Covariance Uncertainty Propagation (CUP).

As CUP is a MC approach, the convergence of the problem should be checked in order to rely on the results after the statistical analysis. The two points where convergence are checked are the same as in the TMC approach: i) the convergence of the cross-section assessing the mean and the rel.std.dev., ii) the convergence of application calculations using the mean and rel.std.dev. values of the output variables.

\subsection{Differences between TMC and CUP}

After describing both approaches to perform MC uncertainty propagation, the differences between them arise.

The first one is related to the sampled variables. In TMC, the nuclear model parameters are sampled, meanwhile in CUP, the cross-sections are directly sampled from the covariance information provided by nuclear data libraries after processing. But both of them have the same aim: produce random nuclear data libraries to be able to perform uncertainty propagation calculations by means of a MC approach.

The next difference is which uncertainty values are being propagated for each cross-section. The TMC approach can use different sources of information to calculate their random files, such as the EXFOR database (experimental data), nuclear data libraries or/and other compilations. These sources can be mixed or used individually. In the other hand, the CUP approach only propagates the uncertainty information of the nuclear data library which is being used.

The third difference is that the TMC approach does not need the neutron spectrum of the application for generating random crosssection libraries, while the CUP approach needs first to process the data, and that means the neutron spectrum is needed before new random libraries are generated.

It can be observed that mean values, their uncertainties and covariance information of each approach could be completely different. But also, they could be completely equivalent if the source of information for TMC was the same as for CUP. If the random cross-section files generated by TMC are condensed in mean values, std.dev. values and covariance matrices by means of performing a statistical analysis of the cross-sections in, for example, 1000 files, a full set of covariance matrices with mean values can be provided as start point of CUP. So, when the CUP approach is carried out, the uncertainty information should be the same as in the TMC approach. Fig. 3 presents the flowchart of using TMC as source of uncertainty information for the CUP approach.

But, it is still possible to find differences because there is at least one assumption in the CUP approach: the chosen PDF is used to sample the cross-section information. Thus, the CUP approach can be partially/totally based on the TMC approach. But, does it mean that both approach will give the same results? and Which information is lost during the condensation process?

\subsection{TMC and CUP applied to activation calculation}

An activation calculation consists in the prediction of isotopic composition and its derived magnitudes such as decay heat, radio-toxicity and activity throughout the burn-up and the cooling time of nuclear fuel. In this case, TMC and CUP will be used to propagate only the cross-section uncertainties.

To perform this kind of calculation, the ACAB code (Sanz et al., 2008) was used. The data needed from the cross-sections is its collapsed value to one-group. There is no need to keep the energy structure because only the reaction rate is used. The reaction rate can be calculated as a cross-section collapsed to one-group multiplied by the total neutron flux. That means also that their uncertainties should be collapsed to one-group. This collapsing process reduces the amount of variables to sample. The collapsing process is done by NJOY which can handle the cross-sections and their uncertainties and collapse to any group-wise structure, also to one-group as activation calculations need.

\subsubsection{TMC on activation calculations}

When TMC is used in activation calculations, it can be split into four stages. The first part is the generation of the required amount of random cross-section libraries, where TASMAN is used. The second one is to process and collapse the cross-section data 


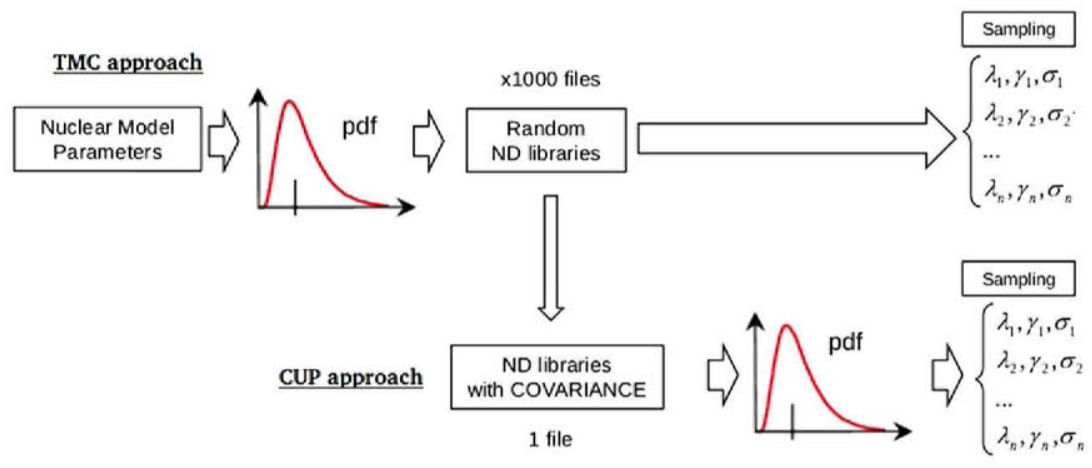

Fig. 3. Flowchart of TMC and CUP "equivalent" calculations.

libraries using NJOY. The third stage is to translate from ENDF-format to ACAB-format. The last stage is to feed ACAB with these random libraries and perform the statistical analysis of the response variables. This process is presented in Fig. 4a.

\subsubsection{CUP on activation calculations}

The scheme of using CUP in activation calculations is quite similar to TMC. The first stage is to process the cross-section data library with their uncertainty information and to collapse both data into one-group. Then, the second stage is to convert from ENDF-format to ACAB-format. The third stage consists in the generation of the random libraries using the ACAB-format library which stores uncertainties by means of random library generator. This generator uses a random number generator to sample the cross-sections and it takes into account the possible cross-correlations (correlations between different reactions) by a Cholesky Decomposition of the full covariance matrix. After sampling an amount of random libraries, the fourth stage is to feed the ACAB code with the random libraries and perform the statistical analysis to calculate the uncertainty on the activation calculations. In Fig. 4b, the flowchart of the CUP approach on activation calculations using the $A C A B$ code is presented.

\section{Ads activation calculation}

The purpose of this application is to answer the previous questions about the differences between TMC and CUP, and to check the

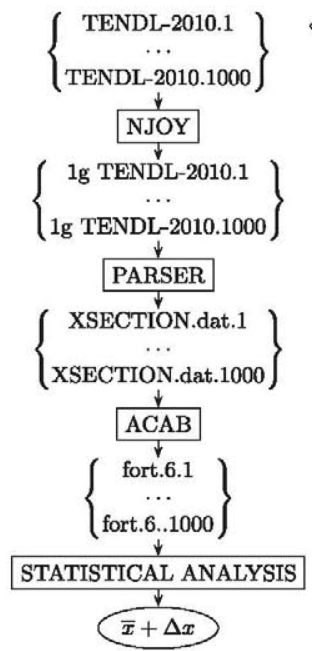

(a) TMC

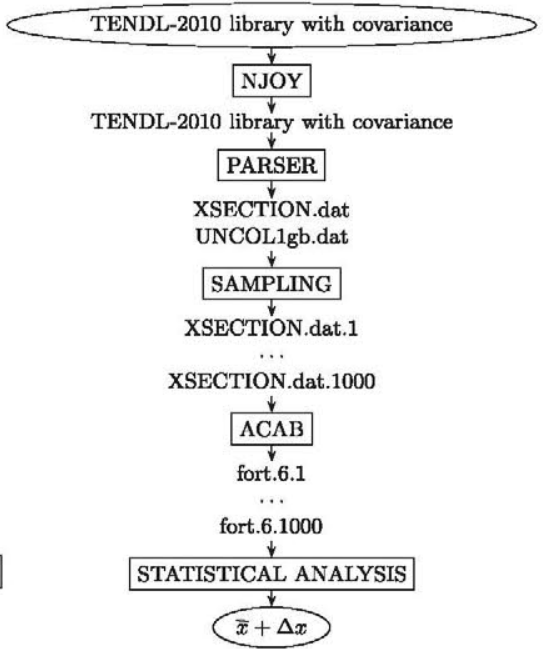

(b) CUP
Fig. 4. Flowcharts of both approaches applied to activation calculations using $A C A B$ code. equivalence of the two approaches in uncertainty propagation calculations for ${ }^{239} \mathrm{Pu}$ and ${ }^{241} \mathrm{Pu}$, when the proper assumptions are done.

In this work, all random cross-section libraries and the cross-section library with covariance information are taken from TENDL2010 for ${ }^{239} \mathrm{Pu}$ and ${ }^{241} \mathrm{Pu}$ and then merged with the complete library of EAF-2010 which contains the cross-sections for all other nuclides.

\subsection{Statement of the problem}

Here, EFIT (Garcia-Herranz et al., 2010) is used as an example of an ADS industrial-scale transmutation facility for uncertainty propagation calculations. Its main characteristics are: core cooled by pure lead, thermal power $400 \mathrm{MW}$, initial total mass of actinides 2.074 tons $(21.7 \% \mathrm{MA})$. One burn-up discharge is studied here, $150 \mathrm{GWd} / \mathrm{tHM}$ ( 778 days). Its neutron spectrum has an average energy of $0.37514 \mathrm{MeV}$ and the total neutron flux is $3.12 \times 10^{15} \mathrm{n} /$ $\left(\mathrm{cm}^{2} \mathrm{~s}\right)$. Its initial fuel composition is given by Garcia-Herranz et al. (2010) and Cabellos et al. (2011).

To perform the calculations using $A C A B$, it is necessary to provide a decay data library and a fission yield data library, in this case the JEFF-3.1.1 library (Kellet et al., 2009) is used in both cases.

The main goal of an ADS facility is to burn transuranic and actinides for reducing their activity during long term cooling time. For this reason, four heavy isotopes are studied: ${ }^{235} \mathrm{U},{ }^{238} \mathrm{U},{ }^{239} \mathrm{Pu}$, ${ }^{241} \mathrm{Pu}$; and one medium mass range isotope: ${ }^{98} \mathrm{Mo}$. But only the results of ${ }^{239} \mathrm{Pu},{ }^{241} \mathrm{Pu}$ are presented in this paper. All these isotopes are in the fuel at the beginning of the cycle (BOC), therefore, they will be transmuted throughout the burn-up.

Both approaches, TMC and CUP, are implemented to propagate the cross-section uncertainties of these isotopes in this application following the flowcharts in Fig. 4a for TMC and Fig. 4b for CUP. The number of atoms throughout the burn-up for the above isotopes are analysed, because all other activation magnitudes are derived from the number of atoms, so their rel.std.dev. should be the same.

\subsection{Setting up the problem}

In order to perform both approaches and to make them equivalent, two assumptions should be done:

1. The first one is the covariance information has to come from the random cross-sections files used in the TMC approach. For this reason, TENDL-2010 is chosen as source of random libraries. It includes the covariance information obtained from the random files. Then, the condensing stage is supposed to be checked and well done. Depending on which isotope is studied, a different amount of random files is used. This amount is enough for cross-sections convergence and for the convergence of the activation calculations. 


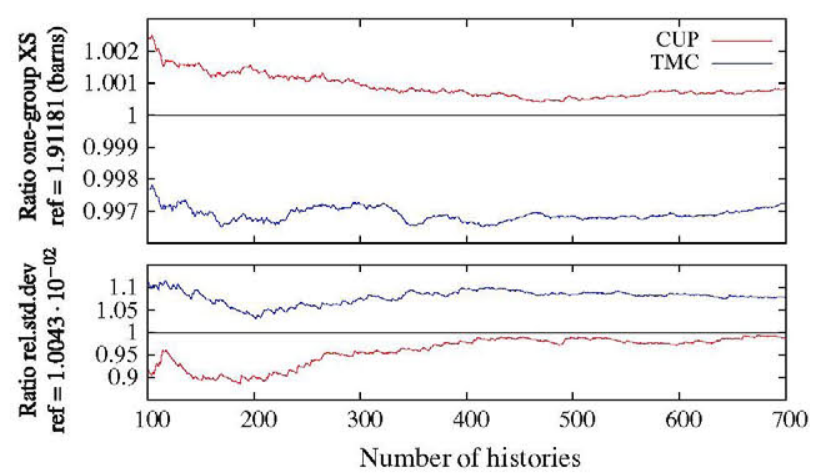

(a) $(n, f)$ cross-section

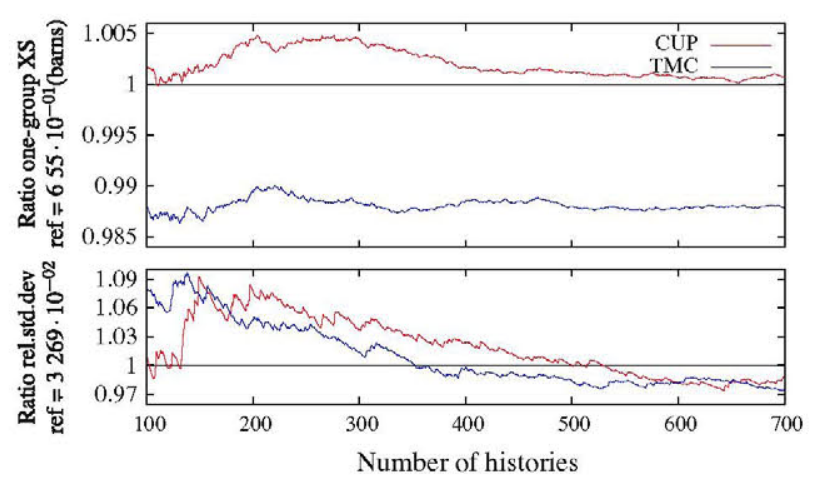

(b) $(n, \gamma)$ cross-section

Fig. 5. Comparison of TMC and CUP one-group cross-section values and their rel.std.dev. as a function of the number of random files for ${ }^{239} \mathrm{Pu}$.

2. The second assumption is which PDF should be chosen for the sampling stage of the CUP approach. As it is mentioned in Section 2.1, TASMAN uses Normal PDFs to sample the nuclear data parameters, so assuming Normal PDFs for CUP approach seems reasonable. But it should be checked that TMC and CUP random libraries follow almost the same PDF.

\subsection{Results and data analysis}

The results for each studied isotope is presented by means of a consistent comparison between the TMC and the CUP approaches. For all figures, blue colour ${ }^{1}$ refers to the TMC approach while red colour refers to the CUP approach, with the only exception of the ratios that are presented in red too.

\subsection{1. ${ }^{239} \mathrm{Pu}$}

For ${ }^{239} \mathrm{Pu}, 700$ random files (TMC) from TENDL-2010 are used, and then, from the cross-section library with covariance information, 700 random cross-section files are obtained using the CUP approach.

After processing all random files, the main cross-sections are compared and showed in Fig. $5(n, f)$ and $(n, \gamma)$. There, the mean value of one-group cross section and its rel.std.dev. are calculated as a function of number of histories. Then, the mean value is divided by the one-group cross-section obtained from processing the TENDL-2010 file with covariance, that means, the cross-section values used as mean value for the CUP approach in the sampling stage. Also, the rel.std.dev. is divided by the rel.std.dev. obtained from processing the TENDL-2010 file with covariances. The mean value and its rel.std.dev. are calculated for TMC and CUP. Here,

\footnotetext{
${ }^{1}$ For interpretation of colour in Figs. 1, 3, 5-14, the reader is referred to the web version of this article.
}

the one-group cross-section values and its uncertainty obtained by the processing of TENDL-2010 with covariances information are considered as reference (this file is unique for this isotope, not like the random files created from it in the CUP approach, or the random files used to create it). These reference values, which are used to divide the mean value and its rel.std.dev., can be read in the label of $Y$-axis.

Because CUP uses Normal PDF based on the reference values, the mean values and their rel.std.dev. of the cross-sections should be close to one, but because of the statistics, deviations appear. When the cross-sections for TMC are analysed, it is observed that:

- In Fig. 5a, the largest ${ }^{239} \mathrm{Pu}$ cross-section $(n, f)$, is presented. It cross-section value is 1.912 (barns) with a rel.std.dev. of $1.0043 \%$. Here, both TMC and CUP mean values are close to the reference (less than $0.3 \%$ of difference); the TMC rel.std.dev. is around $7.5 \%$ greater than the reference while the CUP rel.std.dev. is close to the reference.

- In Fig. 5b, the second largest cross-section, $(n, \gamma)$, is showed. It is equal to $6.550 \times 10^{-1}$ barns, and its rel.std.dev. is $3.269 \%$. Here, the CUP mean value is close to the reference, while the TMC mean value is $1.2 \%$ smaller than the reference. However, their rel.std.dev. are close to each other, around $2 \%$ smaller than the reference.

Then, for the two main reactions: $(n, f)$ and $(n, \gamma)$, random files from TMC and CUP are in close agreement with each other and with the reference values. All the other reactions have a reference mean value less than $10^{-3}$ barns, so they are neglected.

To check that both approaches give the same PDF, Fig. 6 is presented. The black line is the Normal PDF whose area is multiplied by the CUP area in order to represent the PDF that CUP should follow. In Fig. 6a, the reaction $(n, f)$ is showed. There, both PDFs are in close agreement with each other. Fig. $6 \mathrm{~b}$ shows the reaction $(n, \gamma)$, where the PDF of TMC differs from the Normal PDF of CUP, and it looks more like a Log-Normal PDF. Consequently, it leads to different results when the activation calculations are performed, when the PDF of the number of atoms is analysed.

Cross-correlations are not important is this calculation, because the covariance matrix between the main reactions $(n, f)$ and $(n, \gamma)$, presented in Fig. 7b, shows that there are only small correlation between energy groups. The cross-correlation in one group is $3 \times 10^{-3}$, and thus its effect is also negligible, as it can be seen in Fig. 7a, where each point represents a pair of $(n, f)$ and $(n, \gamma)$ cross-sections of the same random library. This clearly indicates that TMC random values are not correlated.

All these random files generated by TMC and CUP are launched on ACAB. As it is described above in Sections 2.1 and 2.2, only the number of atoms during the burn-up is analysed and presented in Fig. 8.

The ratio of mean values of CUP/TMC shows that both values are close to each other and its difference is less than $0.3 \%$ at the end of burn-up. When the rel.std.dev. is observed, it becomes relevant after $10^{7} \mathrm{~s}$ of burn-up for both approaches. The ratio CUP/TMC shows that the CUP rel.std.dev. is $4.8 \%$ greater than TMC. Below $10^{6} \mathrm{~s}$, results are meaningless. The histogram of the number of atoms of ${ }^{239} \mathrm{Pu}$ at the end of the burn-up is presented in Fig. 9. It can be seen that the percentile 95 for TMC is greater than for CUP. That means that the tail of TMC provided greater values than CUP although CUP provides a rel.std.dev. greater than TMC.

The convergence is checked for all time steps as it is shown in Fig. 10 for the end of the burn-up. It can be seen that an increase of the number of histories does not change the average values of the mean value and the rel.std.dev.

This $4.8 \%$ of difference between the rel.std.dev. of TMC and CUP comes from: 


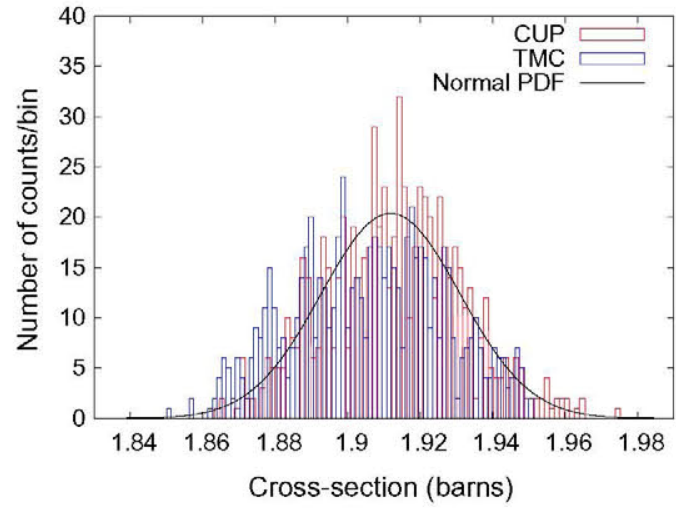

(a) $(n, f)$ cross-section

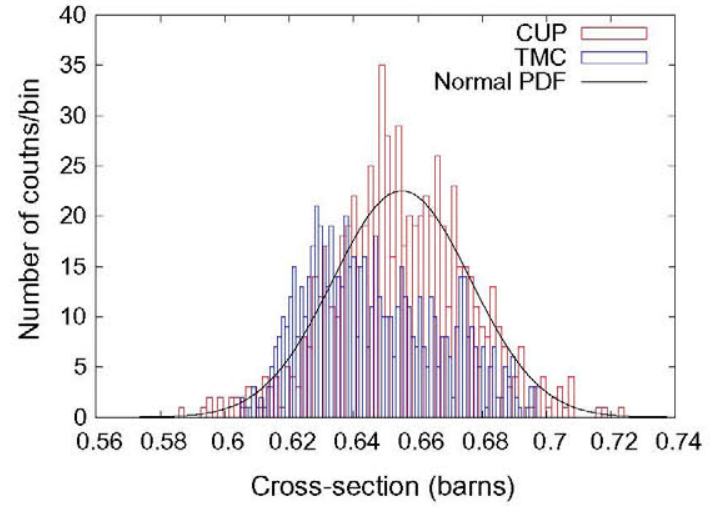

(b) $(n, \gamma)$ cross-section

Fig. 6. One-group cross-section histograms for ${ }^{239} \mathrm{Pu}(n, f)$ and $(n, \gamma)$ reactions, and the Normal PDF generated by covariance information.

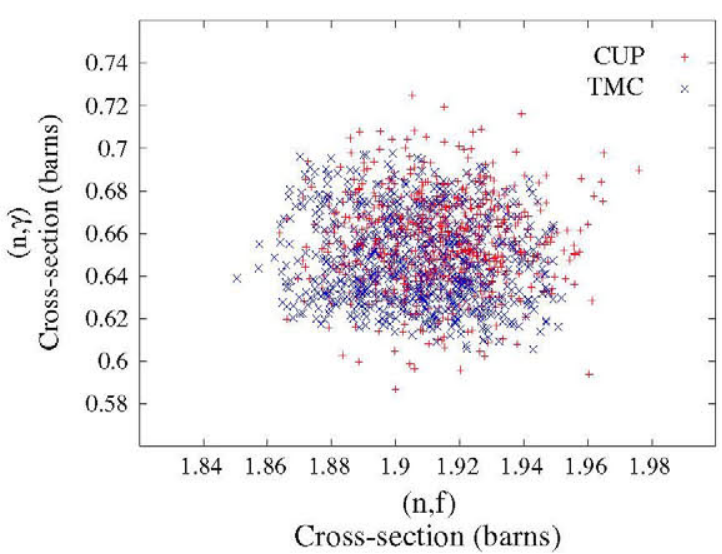

(a) TMC/CUP $(n, f)-(n, \gamma)$ cross-sections

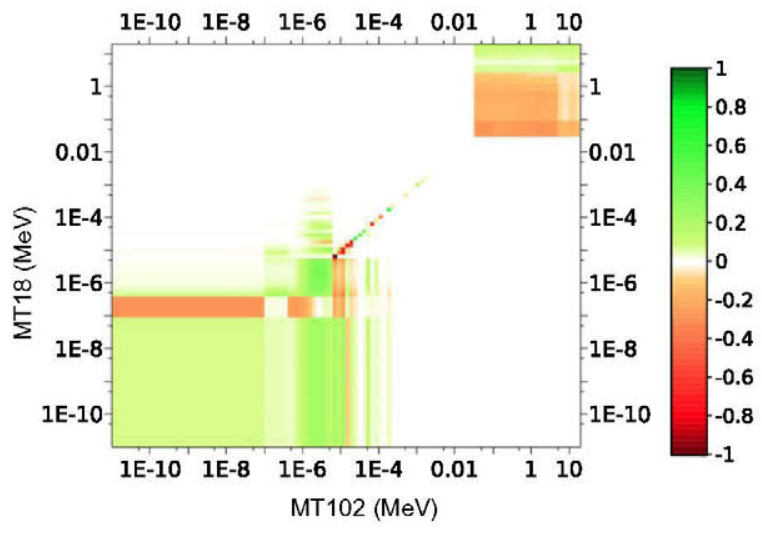

(b) $(n, f)-(n, \gamma)$ correlation matrix

Fig. 7. Comparison of the pair $(n, f)-(n, \gamma)$ cross-section values of TMC and CUP and the correlation matrix in multi-group provided by TENDL-2010 for ${ }^{239}$ Pu.

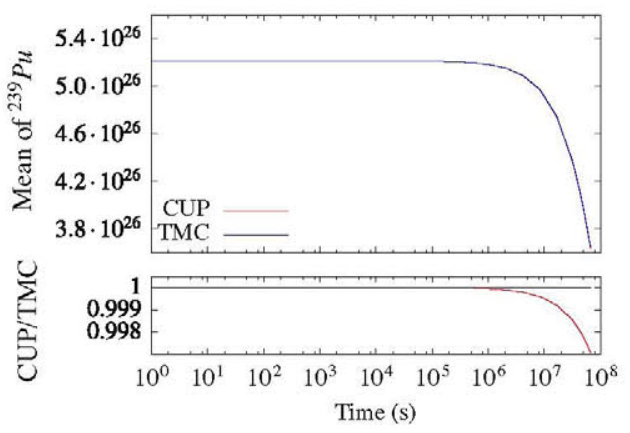

(a) mean value

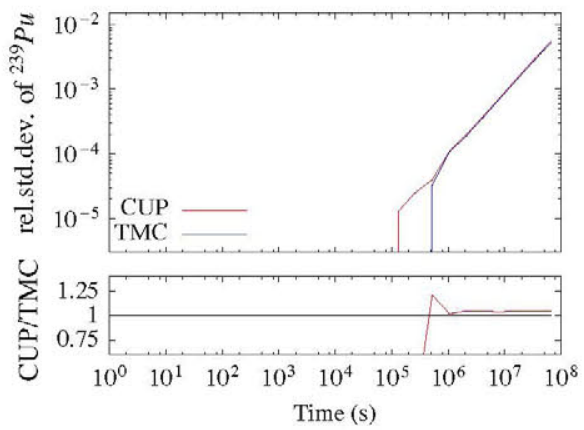

(b) rel.std.dev.

Fig. 8. Mean value, its rel.std.dev. and the ratio CUP/TMC for the number of atoms during burn-up of ${ }^{239} \mathrm{Pu}$.

- The bias between mean values of the cross-sections for each approach, but the most important reactions are in close agreement between each other. Only for $(n, \gamma)$ reaction, the TMC approach provides a mean value $1.2 \%$ smaller than the one provided by the CUP approach.

- The bias between rel.std.dev. of the cross-sections for each approach, especially in $(n, f)$ reaction, where the TMC rel.std.dev. is about $8 \%$ greater than the CUP rel.std.dev.

- The assumption of Normal PDF for the CUP approach does not yield a fair description of the $(n, \gamma)$ cross-section given by TMC.
- The cross-correlations between reactions are not taken into account in the CUP approach, but as it is presented above, the TMC approach does not exhibit cross-correlations among the most important reactions.

- Differences during the condensation process for the generation of the covariance information from the random files of TMC. Instead of using the mean value (the mean value obtained from this amount of random libraries) for calculating the covariance terms, the best-value (which fits best the experimental data) is used. Because of that, a small bias between the CUP mean value and the TMC mean value appears. 


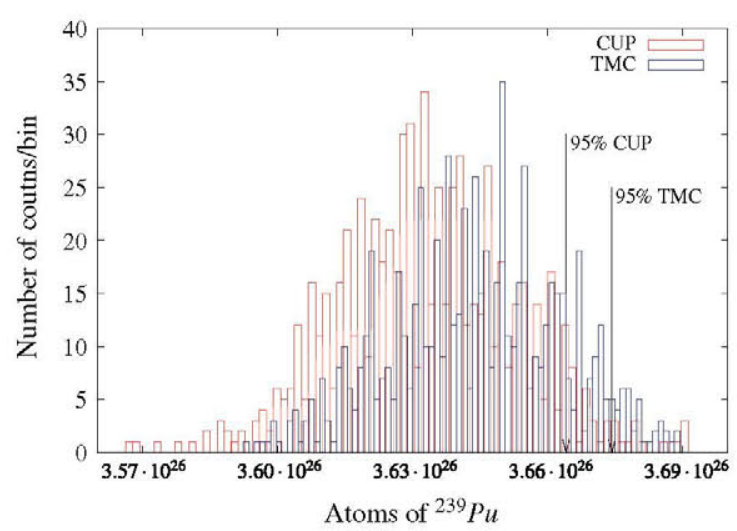

Fig. 9. Histogram of the number of atoms of ${ }^{239} \mathrm{Pu}$ at the end of the burn-up for TMC and CUP.

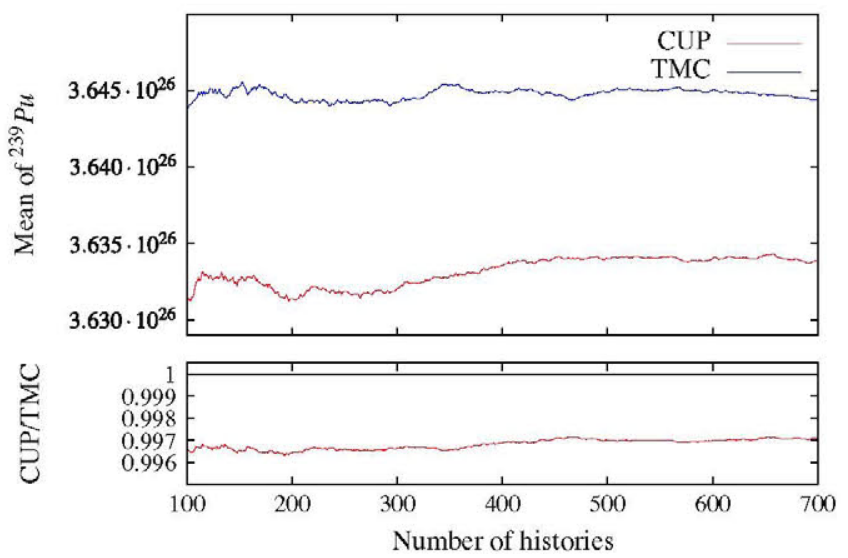

(a) mean value

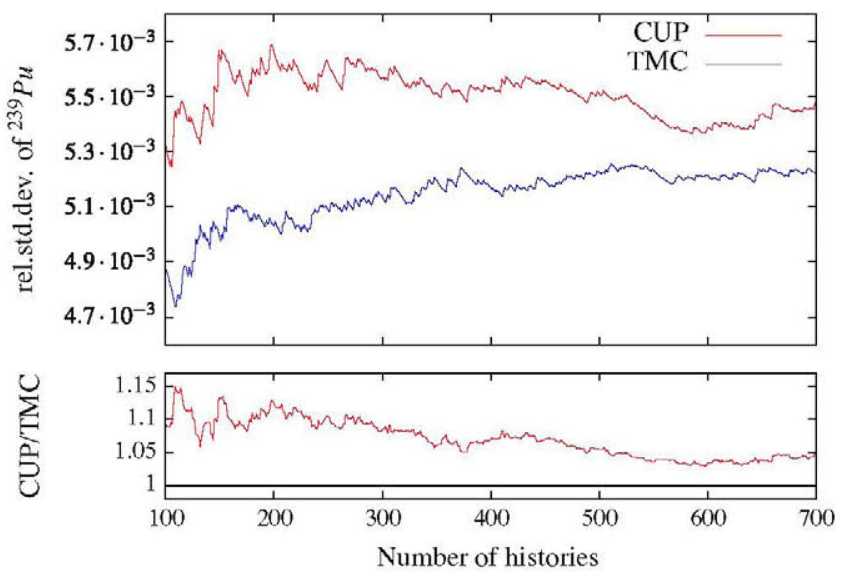

(b) rel.std.dev.

Fig. 10. Mean value, its rel.std.dev. and the ratio CUP/TMC for the number of atoms during the burn-up of ${ }^{239} \mathrm{Pu}$ as a function of the number of histories at the end of burn-up.

\subsection{2. ${ }^{241} P u$}

For ${ }^{241} \mathrm{Pu}$, the implementation of TMC and CUP uses an amount of 700 random libraries for each one.

After processing all random files, and the generation of 700 of random files using the CUP approach, the main reaction cross-sections $(n, f)$ and $(n, \gamma)$, are compared and showed in Fig. 11 as a function of the number of random libraries. The values presented are the ratios when they are divided by the reference values which are the cross-section and the rel.std.dev. of each reaction provided by the TENDL-2010 file with covariances. These values can be read in the $Y$-axis label.

In Fig. 11, differences are found between CUP and TMC:

- In Fig. $11 \mathrm{a}$, the most important reaction of ${ }^{241} \mathrm{Pu}(n, f)$, is presented. It has a cross-section value of 1.53 barns, and its rel.std.dev. value is $3.291 \times 10^{-2}$. For this reaction, TMC and CUP provide mean values which are $1 \%$ smaller than the reference value. Their rel.std.dev. values reach also a good agreement with reference, because TMC provides a rel.std.dev. value $4 \%$ smaller than reference and CUP provides a rel.std.dev. $2 \%$ greater than reference.

- In Fig. $11 \mathrm{~b}$, the second greatest cross-section, $(n, \gamma)$, is presented. Its cross-section value is $3.648 \times 10^{-1}$ barns, with a rel.std.dev. of $1.393 \times 10^{-1}$. Here, TMC provides a mean value $2.5 \%$ greater than reference while CUP provides a mean value far less than $0.5 \%$ smaller than the reference. The rel.std.dev. provided by TMC is $4 \%$ greater than reference and the CUP rel.std.dev. is less than $1 \%$ smaller than reference.

For these two reactions mentioned above, the random files of TMC and CUP do not present great differences between each other.

The histograms of each approach for $(n, f)$ and $(n, \gamma)$ reactions are presented in Fig. 12. The black solid line represents the Normal PDF that the CUP values should follow. In Fig. 12a, the reaction ( $n$, $f$ ) is showed. There, both PDFs are in close agreement with each other, both shapes almost follow the shape of a Normal PDF. Fig. 12b shows the reaction $(n, \gamma)$. The shape of TMC does not follow a Normal PDF. Therefore, it is a source of difference between approaches after the activation calculation.

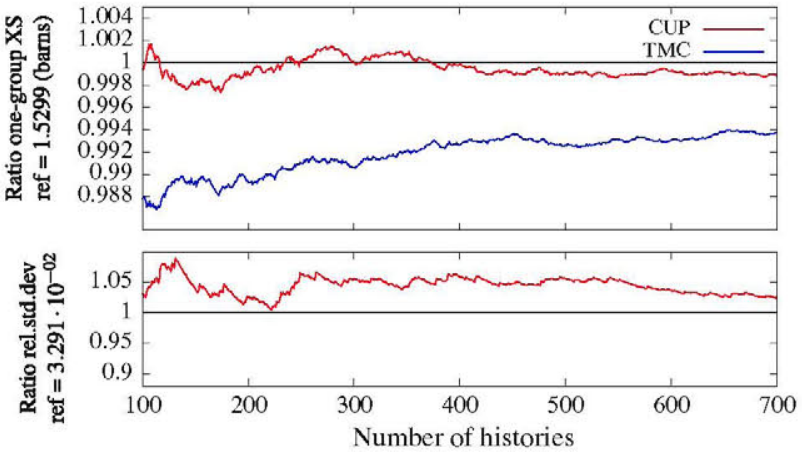

(a) $(n, f)$ cross-section

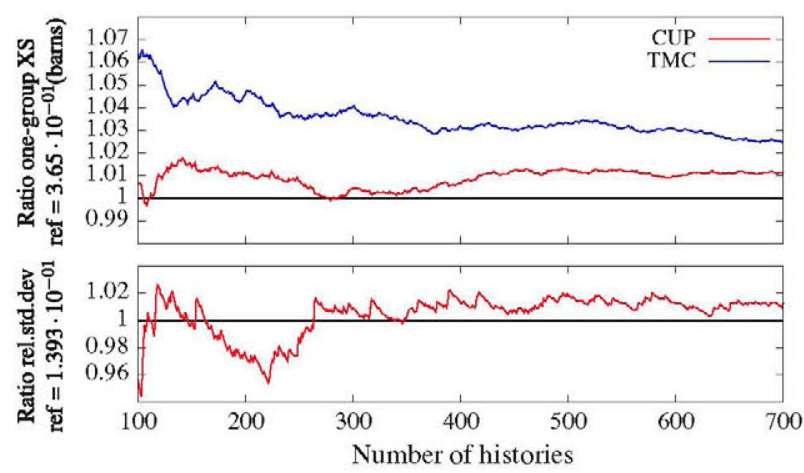

(b) $(n, \gamma)$ cross-section

Fig. 11. Comparison of TMC and CUP one-group cross-section values and their rel.std.dev. as a function of the number of random files for ${ }^{241} \mathrm{Pu}$. 


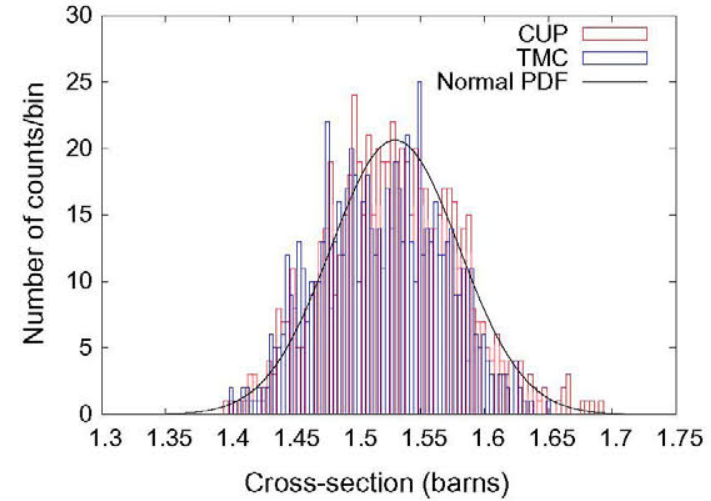

(a) $(n, f)$ cross-section

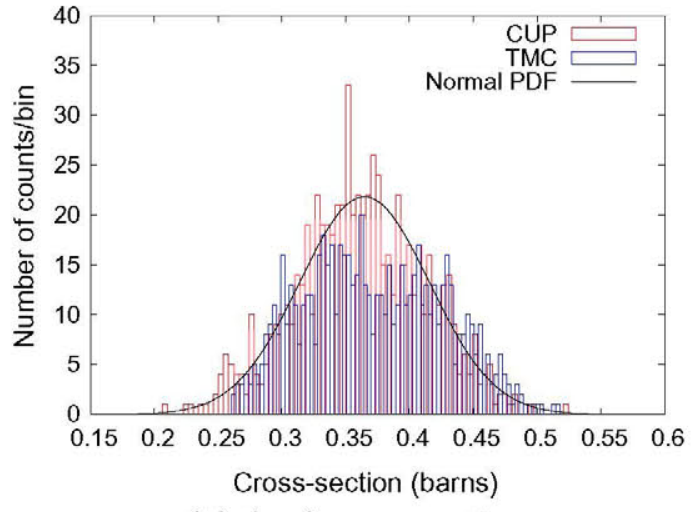

(b) $(n, \gamma)$ cross-section

Fig. 12. One-group cross-section histograms for ${ }^{241} \mathrm{Pu}(n, f)$ and $(n, \gamma)$ reactions, and the Normal PDF generated by covariance information.

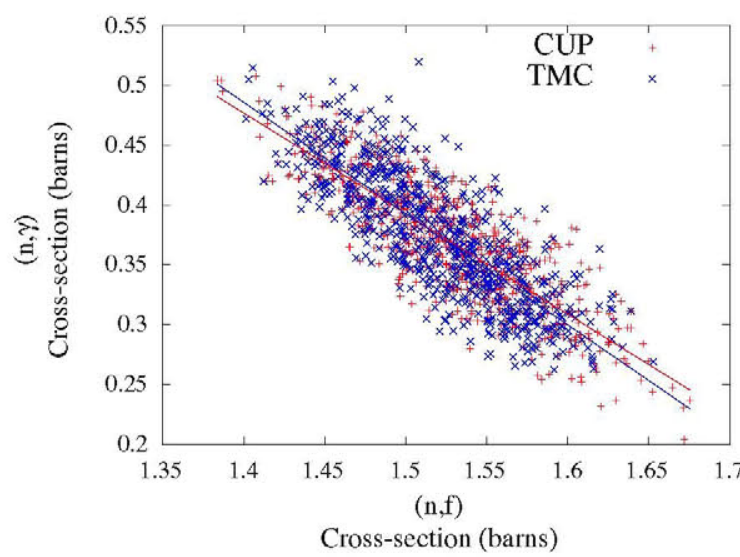

(a) TMC/CUP $(n, f)-(n, \gamma)$ cross-section

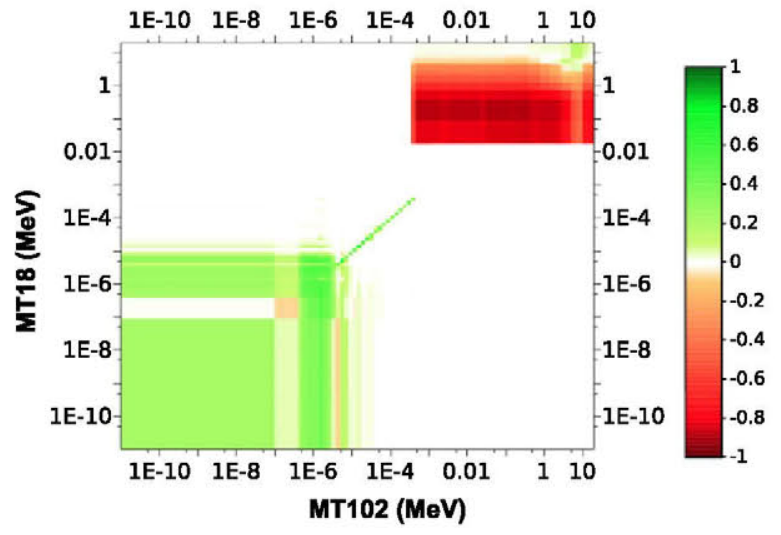

(b) $(n, f)-(n, \gamma)$ correlation matrix

Fig. 13. Comparison of the pair $(n, f)-(n, \gamma)$ cross-section values of TMC and CUP and the correlation matrix in multi-group provided by TENDL-2010 for ${ }^{241} \mathrm{Pu}$.

For this isotope, the cross-correlation matrix of $(n, f)-(n, \gamma)$ cross-sections, presented in Fig. 13b, provided by the library with covariance information shows an anti-correlation between reactions. Using this information in the sampling stage, correlated random numbers can be obtained by using a Cholesky Decomposition. These random cross-section values of $(n, f)$ and $(n, \gamma)$ are compared with the ones provided by TMC in Fig. 13a, where the least-square fittings with linear function for both approaches, CUP and TMC, are presented too. The pairs of the CUP cross-sections provide a correlation factor of -0.83414 . The correlation factor provided by TENDL library is -0.8370 and the correlation factor calculated from TMC cross-sections is -0.81792 . Thus, all values are in close agreement between each other.

After analysing the differences between the cross-sections, $A C A B$ is fed with them. Only the number of atoms during the burn-up is analysed in Fig. 14a. The ratio of mean values of CUP/ TMC shows that both values are the same during the burn-up. When the rel.std.dev. is observed in Fig. 14b, the differences become relevant after $1.05 \times 10^{6} \mathrm{~s}$ of burn-up for both approaches. The ratio CUP/TMC of rel.std.dev. shows that CUP is 0.95 times the TMC value, so a good agreement is reached by both approaches. When the percentiles 95 are compared, both of them are close to each other, and their histograms show that both approaches have the same tails for their distributions.

The mean value and the rel.std.dev. are plotted as a function of number of histories in order to check the convergence as it is done in the previous isotope. The convergence of the mean value and the rel.std.dev. is guaranteed by 700 histories.

In Table 1 , it can be observed that when at least $(n, f)$ and $(n, \gamma)$ uncertainties are propagated together, "All reactions" row, a smaller rel.std.dev. is obtained than when these uncertainties are propagated individually, "a. $(n, f)$ " and "b. $(n, \gamma)$ " rows. So that reveals the importance of the negative cross-correlations between $(n, f)$ and $(n, \gamma)$. Therefore, it has to be taken into account in order to get a CUP approach equivalent to the TMC approach. If not, the CUP value goes up to 2.5 times the TMC rel.std.dev. value instead of being only $5 \%$ smaller than TMC.

Then, the sources of the difference between the rel.std.dev. of TMC and CUP come from:

- The bias between mean values of the cross-sections for each approach, but as it is analysed above, its effect should be small.

- The bias between rel.std.dev. of the cross-sections for each approach, but their differences do not go above 3\% for the most important cross-sections.

- The cross-correlations between $(n, f)$ and $(n, \gamma)$ reactions have to be taken into account in the CUP approach if equivalent results to the TMC approach are wanted.

- During the condensation process for the generation of the covariance information from the random files of TMC, instead of using the mean value (the mean value obtained from this amount of random libraries), the best-value (which fits best 


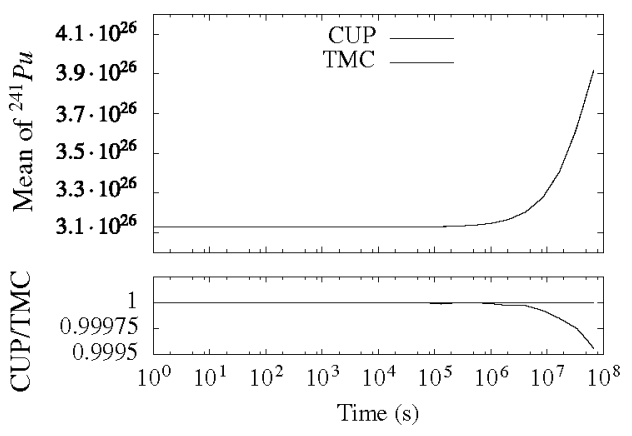

(a) mean value

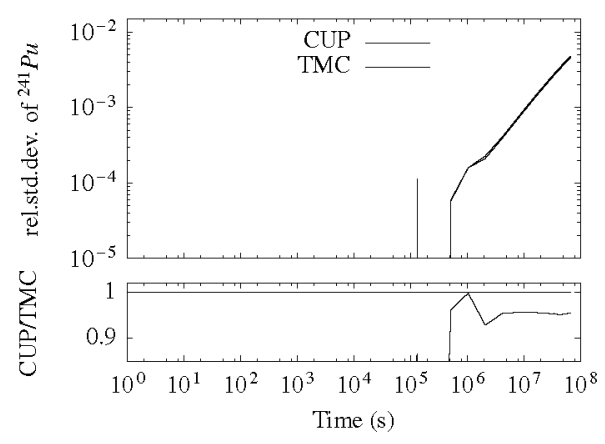

(b) rel.std.dev.

Fig. 14. Mean value, its rel.std.dev. and the ratio CUP/TMC for number of atoms during burn-up of ${ }^{241} \mathrm{Pu}$.

Table 1

Rel.std.dev. values of the number of atoms of ${ }^{241} \mathrm{Pu}$ at the end of the burn-up.

\begin{tabular}{llll}
\hline rel.std.dev. & TMC $(\%)$ & CUP $(\%)$ & CUP without cross-correlation (\%) \\
\hline All reactions & 0.48 & 0.46 & 1.14 \\
a. $(n, f)$ & 0.73 & 0.81 & \\
b. $(n, \gamma)$ & 0.77 & 0.75 & \\
\hline
\end{tabular}

the experimental data) is used. Because of that, a small bias between the CUP mean value and the TMC mean value appears.

\section{Conclusions}

The comparison of two different Monte Carlo approaches for nuclear data uncertainty propagation in activation calculations has been performed. For this purpose, TENDL-2010 libraries have been used.

Two isotopes have been presented here: ${ }^{239} \mathrm{Pu}$ and ${ }^{241} \mathrm{Pu}$. For both isotopes, the differences between CUP and TMC cross-section mean values and their rel.std.dev. propagated in the activation calculations provoke a deviation from TMC rel.std.dev. for the number of atoms during the burn-up for the CUP approach that does not go above $5 \%$. For the case of ${ }^{239} \mathrm{Pu}$, the histogram obtained from the TMC presents a percentile 95 greater than the CUP one, but meanwhile, the rel.std.dev. of CUP is greater than TMC. Great difference between approaches could be found in ${ }^{241} \mathrm{Pu}$ if the cross-correlation between the main reactions were not taken into account, up to 2.5 times.

Thus, regarding the questions presented at Section 1, it can be seen that CUP approach is close to be equivalent to TMC approach, under the proper assumptions: use of the cross-section library with covariance information obtained from the random cross-sections libraries; and choosing a PDF for the cross-section that can represent the PDF cross-section of TMC. But, as it is presented throughout this work, the Normal PDF is not always the best representation for the TMC approach. And such a difference could play an important roll in other frameworks such as safety analysis, where the tails of the PDF for actinides are important for reactivity coefficients or $k_{\text {eff. }}$. Thus, in further studies, different PDFs will be studied and their effects compared, and also in different frameworks in order to check the relevance of choosing one PDF or another. Maybe in a near future, suggestions of which PDF should be used for each cross-section could be provided.

New and better sampling methods are being studied in order to be implemented in the CUP approach, such as Latin Hipercube Sampling (LHS), that will be compared with the "random sampling" method used for sampling cross-section values in this work.

\section{Acknowledgements}

The research leading to these results has received funding from the European Atomic Energy Community's Seventh Framework Programme [FP7/2007-2013] under Grant agreement No. 249671, (FP7-EURATOM-FISSION-2009:Project ANDES/249671).

This work is also partially supported by "Ministerio de Educación (Ministry of Education)" of Spain with the FPU Program for teaching and researching formation (Programa de Formación de Profesorado Universitario) under Grant AP2009-1801 for the first author.

\section{References}

Cabellos, O., Garcia-Herranz, N., de La Obra, C.J.D., Alvarez-Cascos, R., Sanz, J., Ogando, F., Sauvan, P., 2011. Propagation of nuclear data uncertainties in transmutation calculations using ACAB code. J. Korean Phys. Soc. 59 (23), 12681271.

Cacuci, D.G., 2003. Sensitivity and Uncertainty Analysis. Chapman Hall/CRC, London.

Chadwick, M., Obložinskỳ, P., Herman, M., Greene, N., McKnight, R., Smith, D., Young, P., MacFarlane, R., Hale, G., Frankle, S., Kahler, A., Kawano, T., Little, R., Madland, D., Moller, P., Mosteller, R., Page, P., Talou, P., Trellue, H., White, M., Wilson, W., Arcilla, R., Dunford, C., Mughabghab, S., Pritychenko, B., Rochman, D., Sonzogni, A., Lubitz, C., Trumbull, T., Weinman, J., Brown, D., Cullen, D., Heinrichs, D., McNabb, D., Derrien, H., Dunn, M., Larson, N., Leal, L., Carlson, A. Block, R., Briggs, J., Cheng, E., Huria, H., Zerkle, M., Kozier, K., Courcelle, A., Pronyaev, V., van der Marck, S., 2006. ENDF/B-VII.0: next generation evaluated nuclear data library for nuclear science and technology. Nucl. Data Sheets 107 (12), 2931-3060.

Garcia-Herranz, N., Cabellos, O., Âlvarez-Velarde, F., Sanz, J., González-Romero, E.M., Juan, J., 2010. Nuclear data requirements for the ADS conceptual design EFIT: uncertainty and sensitivity study. Ann. Nucl. Energy 37 (11), 1570-1579.

Gentle, J.E., 2003. Random Number Generation and Monte Carlo Methods, second ed. Springer, New York.

Kellet, M., Bersillon, O., Mills, R., 2009. The JEFF-3.1/-3.1.1 Radioactive Decay Data and Fission Yields Sub-libraries.

Koning, A.J., Hilaire, S., Duijvestijn, M., 2009. TALYS-1.2: User Manual.

Koning, A.J., Rochman, D., 2008. Towards sustainable nuclear energy: putting nuclear physics to work. Ann. Nucl. Energy 35 (11), 2024-2030.

Koning, A.J., Rochman, D., 2010. TENDL-2010. <ftp://ftp.nrg.eu/pub/www/talys/ tendl2010/tendl2010.html>.

Koning, A.J., Rochman, D., 2011. Modern nuclear data evaluation: straight from nuclear physics to applications. J. Korean Phys. Soc. 59 (23), 773-778.

MacFarlane, R., Muir, D., 1994. The NJOY Nuclear Data Processing System.

Rochman, D., Koning, A.J., van der Marck, S.C., 2009. Uncertainties for criticalitysafety benchmarks and keff distributions. Ann. Nucl. Energy 36 (6), 810-831.

Rochman, D., Koning, A.J., van der Marck, S.C., 2011. Exact nuclear data uncertainty propagation for fusion design. J. Korean Phys. Soc. 59 (23), 1386-1389.

Rugama, Y. Henriksson, H., Goutte, H., Faust, H., Fioni, G., Goutte, D., 11 May 2005-14 May 2005. NEA nucleat data services: EXFOR, JANIS and the JEFF project. In: 3rd International Workshop on Nuclear Fission and Fission-Product Spectroscopy, vol. 798. Affiliation: OECD/NEA Data Bank, 12 Bld des lles, 92130 Issy-lesMoulineaux, France; Correspondence Address: Rugama, Y.; OECD/NEA Data Bank, 12 Bld des lles, 92130 Issy-les-Moulineaux, France, pp. 61-68, sponsors: CEA/DAM, Bruyeres-le-Chatel; CEA, Cadarache; CEA/DSM, Saclay; ILL, Grenoble; GANIL, Caen; Conseil Regional Provence Alpes-Cote d'Azur.

Sanz, J., Cabellos, O., García-Herranz, N., 2008. ACAB-2008: Activation Abacus Code V2008.

Sublet, J.-C., Packer, L.W., Kopecky, J., Forrest, R.A., Koning, A.J., Rochman, D., 2010. The European Activation File: EAF-2010 Neutron-induced Cross Section Library. 